\title{
金属材料热处理变形的影响因素与控制策略
}

\author{
韩永波 \\ 通标标准技术服务 (青岛)有限公司，山东青岛 260000
}

\begin{abstract}
[摘要] 近年来, 在社会经济水平不断提升的带动下, 使得我国科学技术的发展取得了良好的成绩。在这种形势下人们对金属 材料的研究工作越发的重视, 就金属材料来说, 其中热处理是其中最为关键的一项内容, 这一工艺往往都是被人们引用在对 金属材料所实施的特殊处理工序之中, 其作用就是能够从根本上提升整个金属材料的力学性质, 为金属材料的后续使用效果 的提升创造良好的基础。就现如今各方面实际情况来说，尽管金属材料热处理工艺的发展历经了几十年的实践，从某种层面 上已经能够满足我国实际生产工作对金属材料的需要, 但是因为在整个过程中极易发生形变的情况, 所以会对金属材料在使 用过程中的作用的发挥起到一定的限制。
\end{abstract}

[关键词]金属材料; 热处理; 变形; 影响因素; 控制

DOI: $10.33142 / a e m . v 2 i 5.2142$ 中图分类号: TG156 文献标识码：A

\section{Influencing Factors and Control Strategies of Metal Material Heat Treatment Deformation}

HAN Yongbo

SGS-CSTC (Qingdao), Qingdao, Shandong, 260000, China

\begin{abstract}
In recent years, driven by the continuous improvement of social and economic level, the development of science and technology in China has made good achievements. In this situation, people pay more and more attention to the research work of metal materials. In terms of metal materials, heat treatment is one of the most critical contents. This process is often cited in the special treatment process of metal materials. Its role is to fundamentally improve the mechanical properties of the whole metal materials, and create a good foundation for the subsequent use effect of metal materials. As far as the actual situation is concerned, although the development of metal material heat treatment process has gone through several decades of practice, to some extent, it can meet the needs of China's actual production work for metal materials, but because of the deformation in the whole process, it will play a certain limit on the role of metal materials in the use process.
\end{abstract}

Keywords: metal materials; heat treatment; deformation; influencing factors; control

\section{引言}

切实的运用热处理技术来针对金属材料实施加工处理，不但可以有效的增强金属材料的综合性能，并且可以推动 相关领域的稳步发展, 将金属材料所拥有的关键影响作用更好的施展出来。但是在实际开展热处理加工工作的时候, 经常会受到外界多种因素的影响, 从而会对工作的有序开展造成一定的制约, 这样对于金属材料的发展是会形成一定 的阻碍。鉴于此, 这篇文章围绕金属材料热处理变形影响因素实施深入的研究分析, 并针对引发金属材料形变的根源 提出了针对性的解决方案，希望能够对金属材料处理行业的稳步健康发展有所帮助。

\section{1 金属材料热处理变形的影响因素}

\section{1 应力状态影响因素}

在利用专业的技术和方法对金属材料实施热处理加工工作的时候, 因为金属材料自身具有一定的特殊性, 再加上 外界各种因素的影响, 所以导致金属材料的冷热分布存在严重的不均衡的情况, 这样就会对热处理加工工序的顺利实 施造成一定的困扰。在针对金属材料实施热处理加热以及保温错时的时候, 也会受到环境温度的影响, 导致金属材料 内部应力会发生相应的改变, 最终就会导致材料变形的情况。通常情况下, 如果金属材料存在严重的应力分布不均衡 的问题，那么其发生变形的情况的概率是较高的，并且也会对人们判断金属材料的质量产生不良影响。

\section{2 预处理因素}

就当下实际情况来说, 使用最为频繁的预处理方法就是正火处理方法, 将这种方法加以实践运用, 能够有效的清 除盈利, 但是因为会受到环境因素的影响, 所以会在实施正火处理工作的时候, 冷却通常都是自然冷却的形式, 这样 
就会对材料在加热炉中的冷却效果产生一定的限制, 最终发生冷热不均的问题。这类材料在热处理环节中发生变形的 概率较高, 如果不能利用有效的方法来实施正火, 那么也会造成变形概率的提升。

\section{3 淬火介质影响因素}

在针对金属材料实施热处理加工的时候，淬火介质与处理效果存在密切的关联，并且也是引发金属材料变形的根 本因素, 为了从根本上避免金属材料出现变形的情况, 那么就需要充分的结合各方面实际情况来对淬火介质进行恰当 的挑选, 并且要对金属材料质量问题给予重点关注, 保证淬火的效果。其次, 需要严格遵照规范标准要求对介质的摚 拌速度进行全面的控制, 提升搅拌工作的整体效果, 如果戒指搅拌的效率和方法不能满足实际的需要, 那么就会引发 金属材料形变的情况。

\section{2 金属材料热处理变形控制原则}

\section{1 易操作原则}

要想从根本上促进金属材料热处理工艺整体水平的提升, 首先我们需要对加大力度对原材料变形问题进行深入的 研究, 并针对性总结出预防和解决方案, 有效的规避金属材料出现变形的问题。其次, 在针对金属材料的变形问题加 以解决的时候, 需要综合各方面实际情况对变形控制流程实施简化, 确保金属材料变形预控工作的效率和效果得到全 面的提升。

\section{2 实用性原则}

金属材料热处理属于一种最为基本的自然变化过程，在整个自然环境的发展中起到了十分重要的影响作用。在将 金属材料热处理工艺加以实践运用的时候，所需要的金属材料的种类较多，并且技术成本在整体成本中的占比较大。

\section{3 科学性原则}

综合各方面实际情况利用有效的方式方法对金属材料热变形实施全面的管控, 在正式开始操作之前, 需要针对引 发金属材料形变的各类因素进行预判，制定针对性的预防和解决方案，保证工作的科学性。

\section{3 改善金属材料热处理变形的控制措施}

总的来说，在科学技术飞速发展的带动下，使得我国金属热处理工艺的整体水平也不断的提升，并且其所适用的 范围也在不断的扩展。怎样结合现实需要来对现有金属热处理工艺进行优化和创新是当前我国金属材料处理研究工作 人员最为关心的问题。所以我们可以从下面几个方面入手来对金属热处理工艺进行完善和创新，从而有效的规避金属 在热处理过程中发生变形的情况。

\section{1 加强对预处理变形的控制与管理}

要想从根本上控制金属材料热处理工序中金属材料变形的概率，那么最为重要的就是需要综合各方面实际情况， 利用专业的方式方法对温正火材料科学处理, 这样不但可以有效的提升金属材料的性能, 并且还可以规避材料热处理 不均衡的问题发生，从而实现控制金属材料变形概率的目的。因为各类金属材料的性质存在明显的差别，所以各类材 料的结构特征都是不一样的, 所以在开展热处理需要充分结合金属材料的性质来制定针对性的热处理工序，这样才能 保证达到良好的效果，尽可能的规避金属材料变形的情况发生。加大力度针对预处理变形实施全面的把控，尽管这样 做能够有效的提升热处理的效率和效果, 但是在整体成本方面也会有所增加, 所以我们需要切实的综合金属材料情况 来挑选恰当的处理模式。

\section{2 优化原有的淬火处理工艺}

淬火热处理是现如今我国金属材料热处理工艺中最为关键的处理方式, 在整个过程中牵涉到诸多影响因素, 如果 不能合理的进行管控, 那么就会导致金属材料内部作用力的波动, 最终会导致金属材料出现变形的情况, 所以为了能 够尽可能的规避各类因素所导致的金属材料变形的问题，需要专业人员恰当的对淬火处理工艺进行运用，这样才能切 实的控制金属材料发生变形的情况。诸如: 在实际运用淬火热处理工艺进行金属材料加工的时候, 一般来说需要运用 有效的方式方法来对温度实施有效的控制, 实践不能过短也不能过长, 不然会导致金属受热不均衡的问题, 最终就会 引发金属变形的不良后果。 


\section{3 合理选择冷却方法}

经过对以往大量的金属材料热处理进行综合分析我们发现，通常使用较为频繁的热处理方法为分级淬火和单液淬 火, 其中单液淬火操作起来相对较为简单, 但是实践运用效果较差, 并且引发金属材料发生变形情况的概率较高, 所 以如果条件允许可以将单液淬火调整为分级淬火的方式, 这样才能在较短的时间内将温度较高的金属材料进行快速降 温, 随后利用慢速淬火降温的方法，逐渐的将金属材料的温度进行控制，并达到既定的要求。

\section{4 采用机械化加工技术}

针对金属材料实施热处理的时候，工序的顺序具有一定的固定性，但是因为所加工的金属材料的性质存在明显的 差别, 所以导致针对各类不同性质的金属制定的热处理工艺的工序会存在一定的差异。对于大部分金属材料加工工作 来说, 热处理是较为重要的一个工序, 但是因为一些金属材料具有一定的特殊性, 所以热处理工序的效果往往会受到 金属材料的影响。机械加工处理在针对余量处理方面效果较好，一般情况下，都会对加工工序制定一定的金属变形范 围, 对于完成加工处理的金属材料, 如果出现超出形变规定范围的情况, 那么就需要判断引发变形的主要根源, 从而 选择最佳的处理方法, 从根本上解决变形的问题。 ${ }^{[4]}$

\section{4 结语}

总的来说，近年来我国金属材料热处理研究工作得到了全面的发展，从而使得人们对影响金属热处理效果的各类 因素得以全面的认识，这样才能在进行金属材料热处理的时候有效的对各类因素造成的不良影响进行把控，提升金属 热处理的效果。

\section{[参考文献]}

[1]赵娇娇. 金属材料热处理变形的影响因素与控制策略 $[\mathrm{J}]$. 建筑工程技术与设计,2018(34): 4137 .

[2] 钱文勇, 钱宏义, 周炳欣. 金属材料热处理变形的影响因素与控制策略 [J]。军民两用技术与产品, 2018 (22) : 189-203.

[3]张发海. 金属材料热处理变形的影响因素与控制策略 [J].现代制造技术与装备, 2018 (10) : 146-150.

[4]胡娟. 金属材料热处理变形的影响因素与控制策略 [J]. 建筑工程技术与设计, 2018 (25): 3784 .

作者简介: 韩永波 (1982.5.14-), 男, 毕业于烟台大学材料科学与工程专业, 本科学历, 现在就职于通标标注技术服 务 (青岛) 有限公司, NDT工程师就职了 14 年。 